\section{BREASTFEEDING AND CHILDHOOD OVERWEIGHT}

\author{
N. Ferraria ${ }^{1}$, V. Rodrigues ${ }^{2}$, L. Macedo ${ }^{3}$
}

${ }^{1}$ Pediatric Department, Hospital Nossa Senhora do Rosário, Centro Hopitalar Barreiro- Montijo,

Barreiro, ${ }^{2}$ Hospital Dona Estefânia, Centro

Hospitalar Lisboa Central, ${ }^{3}$ Centro de Saúde da Alameda, Lisboa, Portugal

Background and aims: Childhood obesity and overweight predict short and long-term morbidity as well as obesity in adulthood. Evidence is not consistent about the association of breastfeeding and childhood overweight.

This study aims to evaluate the impact of breastfeeding on the risk of overweight and obesity in school-aged children.

Methods: Cross-sectional study with 125 children, aged 5 to 10 years old, attending a primary health care center in Lisbon.

Height and weight were measured during routine consultation. Other variables were collected based on questionnaires, such as breastfeeding duration, birth weight, diet, lifestyle factors, mother body mass index (BMI) and education. Overweight and obesity were defined as BMI per age equal or above the $85^{\text {th }}$ percentile based on the 2000 Centers for Disease Control and Prevention growth charts.

Results: Prevalence of overweight and obesity was $23,2 \%$. Among the 125 children, 25,6\% were never breastfed and only $28,8 \%$ were breastfed for 6 or more months.

A dose-response effect was identified between the duration of breastfeeding and the prevalence of overweight and obesity: prevalence was 5,6\% for 1 month of exclusive breastfeeding, $4 \%$ for $1-2$ months, $2,4 \%$ for $3-5$ months, $0,8 \%$ for $6-11$ months, and $0 \%$ for 12 or more months. After adjusting for other variables, breastfeeding remained a significant protective factor against the development of overweight.

Conclusion: In our study prolonged breastfeeding is associated with a reduced risk of overweight. These results emphasize the importance and possible preventive potencial of early nutrition in childhood overweight.

\section{POSTNATAL EARLY CATCH-UP GROWTH AND INSULIN SENSITIVITY IN SMALL FOR GESTATIONAL AGE INFANTS.}

\author{
X. Wang, Y. Cui, T. Han \\ Department of Pediatrics, Peking University Third \\ Hospital, Beijing, China
}

Objective: To explore the relationship of postnatal catch-up growth and insulin sensitivity.

Methods: Fasting glucose, insulin and HOMA value in 44 small for gestational age (SGA) infants and 74 appropriate for gestational age (AGA) infants at age 3 month were studied by a prospective study, and the relations with catch up growth were analyzed in 44 SGA infants.

Results: SGA infants had significantly higher fasting insulin concentration and HOMA value than did AGA infants. Adjustment for gestation, there was a negative association between velocity of catch-up growth and insulin concentration and HOMA value in SGA infants $(P<0.05) .12$ non-catch-up growth in weight and length infants had significantly higher fasting insulin concentration and HOMA value than did they on the third day after birth in SGA group respectively [ insulin : $(21.92 \pm 14.25) \mathrm{mU} / \mathrm{L}$ vs (11. $08 \pm 9.59) \mathrm{mU} / \mathrm{L} P<0.05$; HOMA : $(4.15 \pm 2.96)$ vs $(1.86 \pm 1.71), P<0.05]$.

Conclusions: Catch-up growth is closely related with insulin sensitivity in early postnatal life, and poor growth might result in worse insulin sensitivity in SGA infant $s$ during that time.

412

\section{PREVALENCE AND CLINICAL FEATURES OF METABOLIC SYNDROME IN OVERWEIGHT AND OBESE CHILDREN}

\section{A. Macari, L. Papini, M.E. Liverani, A.C. Massolo, F. Cassiani, D. Chialant, M.P. Villa \\ Pediatrics Sant' Andrea Hospital, University 'La Sapienza', II Faculty of Medicine, Rome, Italy}

Aim of the study: To evaluate the prevalence of metabolic syndrome (MetS) and its clinical and biochemical features in an overweight or obese pediatric population.

Methods: 202 consecutive subjects ( M/F 94/108; mean age $10.9 \pm 2.6$ years, range $4-16$ years) with overweight or obesity according to $\mathrm{WHO}$ definition 